\title{
Estado periodontal y microbiota del surco gingival en dentición temporal y mixta. Revisión sistemática*
}

Periodontal Status and Gingival Sulcus Microbiota in Temporary and Mixed Dentition.

Systematic Review

Estado periodontal e microbiota do sulco gengival em dentição temporária e mista.

Revisão sistemática

Fecha de recepción: 19-02-2020 | Fecha de aceptación: 08-06-2020

MARÍA CAMILA QUERUBÍN JIMÉNEZ

Pontificia Universidad Javeriana, Bogotá, Colombia.

querubinmaria@javeriana.edu.co; https://orcid.org/0000-0002-0026-5047

YADIRA SELENNE VELA PALOMINO

Pontificia Universidad Javeriana, Bogotá, Colombia.

selennevela@hotmail.com; https://orcid.org/0000-0002-5013-6251

\author{
MARIO RAFAEL ROMERO SÁNCHEZ \\ Pontificia Universidad Javeriana, Bogotá, Colombia. \\ romero.mario@javeriana.edu.co; https://orcid.org/0000-0003-0927-2371
}


MARÍA MARCELA COLMENARES MILLÁN

Pontificia Universidad Javeriana, Bogotá, Colombia.

colmenaresm@javeriana.edu.co; https://orcid.org/0000-0002-3893-6651

\title{
DABEIBA ADRIANA GARCÍA ROBAYO
}

Pontificia Universidad Javeriana, Bogotá, Colombia.

garciad@javeriana.edu.co; https://orcid.org/0000-0003-0770-9138

\author{
ÁNGELA SUÁREZ CASTILLO \\ Pontificia Universidad Javeriana, Bogotá, Colombia. \\ angelasuarez@javeriana.edu.co; https://orcid.org/0000-0002-5575-5836
}

*Revisión sistemática de la literatura

Correspondencia: querubinmaria@javeriana.edu.co; selennevela@ hotmail.com;

romero.mario@javeriana.edu.co; colmenaresm@javeriana.edu.co; garciad@ javeriana.edu.co

doi: https://doi.org/10.11144/Javeriana.uo39.epms

Cómo citar: Querubín Jiménez MC, Vela Palomino YS, Romero Sánchez MR, Colmenares Millán MM, García Robayo DA. Estado periodontal y microbiota del surco gingival en dentición temporal y mixta. Revisión sistemática. Univ Odontol. 2020; 39. https://doi.org/10.11144/Javeriana.uo39.epms 


\section{RESUMEN}

Antecedentes: Los microorganismos de la biopelícula dental coexisten en relativa armonía. Los cambios microambientales pueden generar un crecimiento excesivo de microorganismos patógenos relacionados con procesos inflamatorios crónicos como gingivitis y periodontitis. La microbiota del surco gingival puede variar sustancialmente dependiendo de las características fisiológicas de niños y adolescentes. Objetivo: Analizar la evidencia sobre la composición bacteriana en niños de 3-12 años y su asociación con el estado gingival y periodontal. Métodos: Se realizó una revisión sistemática mediante una búsqueda en las bases PubMed, SciELO, LiLACS, ProQuest, EBSCO, Web of Science, Scopus y ScienceDirect. Se leyeron títulos, resúmenes y textos completos. Se determinó la calidad y validez de los estudios por medio de las listas Consort y Strobe. Se clasificaron los artículos según nivel de evidencia y grado de recomendación mediante la SIGN. Resultados: Los perfiles microbianos de la biopelícula supra y subgingival en niños son similares en más del $80 \%$ con predominio de Actynomices spp y Streptoccoccus spp, principalmente $S$. sanguinis. La colonización temprana de la microbiota supra y subgingival se relaciona con la presencia de inflamación gingival en niños de mayor edad. La evidencia sobre condición sistémica, características sociodemográficas, interacción bacteriana, composición microbiana y estado periodontal fue limitada y no concluyente. Conclusión: No existe suficiente evidencia que confirme la relación entre la composición bacteriana con el estado gingival y periodontal en niños de 3-12 años. No obstante, se percibe que, a medida que incrementa la edad, se favorece la presencia de patógenos relacionados con la enfermedad periodontal. 


\section{Palabras clave}

bacterias; biopelícula dental; dentición mixta; dentición primaria; gingival; microbiología oral; microbioma; microbiota oral; microorganismos; niño/a; odontología; odontopediatría; patógenos; periodoncia; periodontal; placa dental; subgingival; supragingival

\section{ABSTRACT}

Background: Microorganisms of dental biofilm coexist in relative harmony. Microenvironment changes can result in an excessive growth of pathogenic microorganisms associated with chronic inflammatory processes such as gingivitis and periodontitis. The microbiota of the gingival sulcus can vary substantially depending on physiological characteristics of children and adolescents. Purpose : To analyze the evidence on bacterial composition in 3-to-12-yearold children old and its association with the gingival and periodontal status. Methods: A systematic literature review was conducted using the PubMed, SciELO, LiLACS, ProQuest, EBSCO, Web of Science, Scopus, and ScienceDirect databases. Titles, abstracts, and full articles were read. Quality and validity of studies were determined through the Consort and Strobe checklists. The articles were classified regarding level of evidence and the grades of recommendation through the SIGN. Results: Microbial profiles of supra and subgingival biofilm in children are similar in more than $80 \%$, with predominance of Actinomyces spp. and Streptococcus spp., mainly S. sanguinis. Early colonization of supra and subgingival microbiota was related with the presence of gingivitis in older children. Evidence about general health condition, sociodemographic situation, bacterial interaction, microbial composition, and 
periodontal state was limited and inconclusive. Conclusion: There is not enough scientific evidence to confirm the relationship between bacterial composition and gingival and periodontal status in children aged 3-12 years. However, it is suggested that, as age increases, the presence of periodontal pathogens related to periodontal disease is likely.

\section{Keywords}

bacteria; biofilm; child; dental plaque; dentistry; gingival; microbiome; microorganisms; mixed dentition; oral microbiology; oral microbiota; pathogens; pediatric dentistry; periodontal; periodontics; primary dentition; subgingival; supragingival

\section{RESUMO}

Antecedentes: Os microrganismos do biofilme dentário coexistem em relativa harmonia. Alterações microambientais podem gerar crescimento excessivo de microrganismos patogênicos relacionados a processos inflamatórios crônicos, como gengivite e periodontite. A microbiota do sulco gengival pode variar substancialmente, dependendo das características fisiológicas de crianças e adolescentes. Objetivo: Analisar as evidências sobre a composição bacteriana em crianças de 3 a 12 anos e sua associação com o estado gengival e periodontal. Métodos: Foi realizada uma revisão sistemática por meio de busca nas bases de dados PubMed, SciELO, LiLACS, ProQuest, EBSCO, Web of Science, Scopus e ScienceDirect. Foram lidos títulos, resumos e textos completos. A qualidade e validade dos estudos foi determinada usando as listas Consort e Strobe. Os artigos foram classificados quanto ao nível de evidência e grau de recomendação por meio do SIGN. Resultados: Os perfis microbianos do biofilme supra e subgengival em crianças são semelhantes em mais de 80\% com predomínio de Actynomices spp. 
e Streptoccoccus spp, principalmente $S$. sanguinis. A colonização precoce da microbiota supra e subgengival está relacionada à presença de inflamação gengival em crianças maiores. As evidências sobre a condição sistêmica, características sociodemográficas, interação bacteriana, composição microbiana e estado periodontal foram limitadas e inconclusivas. Conclusão: Não há evidências suficientes para confirmar a relação entre a composição bacteriana com o estado gengival e periodontal em crianças de 3 a 12 anos. Porém, percebe-se que, à medida que a idade aumenta, a presença de patógenos relacionados à doença periodontal é favorecida.

\section{Palavras-chave}

bactérias; biofilme dentário; dentição mista; dentição decídua; gengival; microbiologia oral; microbioma; microbiota oral; microorganismos; menino/a; odontologia; odontopediatria; patógenos; periodontia; periodontal; placa dentária; subgengival; supragengival

\section{INTRODUCCIÓN}

El microbioma oral está influenciado por diferentes factores como la erupción de los dientes temporales y el recambio de la dentición temporal por la dentición mixta. Ello proporciona superficies y sitios de unión que permiten que las bacterias colonicen y afecten los tejidos dentales y los tejidos periodontales (1). Los microorganismos colonizadores primarios tienen acceso a los tejidos periodontales sanos. Sin embargo, la persistencia de biopelícula en el margen y el surco gingival conlleva al desarrollo de la gingivitis, condición reversible que en

algunas personas puede progresar a periodontitis, en especial si estos presentan alguna condición sistémica predisponente $(2,3)$. Se considera a la periodontitis una enfermedad 
infecciosa e inflamatoria que, de acuerdo con el grado de compromiso, puede llevar a la pérdida parcial o total de los tejidos de soporte del diente (4).

La gingivitis es más frecuente entre los niños y adolescentes y se caracteriza por no comprometer la inserción del tejido conectivo; por el contrario, la población adulta se ve afectada con mayor frecuencia por periodontitis que se caracteriza por la pérdida de los tejidos de soporte del diente $(5,6)$. La prevalencia de la enfermedad gingival y periodontal difiere sustancialmente entre niños y adultos. Albandar y Rams (7) describen una prevalencia de gingivitis mayor al $70 \%$ en niños mayores de 7 años, aunque la prevalencia de enfermedad periodontal es baja 0,9-4,5\%. Esto resulta de interés en la investigación odontológica, si se tiene en cuenta el cambio del comportamiento de los microorganismos durante el recambio dental $(8,9)$. En Latinoamérica, la presencia de gingivitis en la población pediátrica oscila entre el 20,6\% y el $90 \%$, mientras que la prevalencia de periodontitis puede afectar aproximadamente al $10 \%$ de los niños y adolescentes (10).

En Brasil, se ha reportado que la gingivitis en niños y adolescentes afecta un $90 \%$ de la población, en tanto que la periodontitis agresiva tiene una frecuencia del 2,1\%. En Argentina, la gingivitis en niños de 4 a 16 años se presenta del $13 \%$ al $70 \%$ y la periodontitis en niños de 7 a 12 años en el $1 \%$. En Venezuela, la gingivitis se ha descrito entre el $40 \%$ y el $60 \%$ en niños de 6 a 9 años, mientras que en Chile aumenta según la edad y varía del 2,6\% al $66 \%$ en niños de 2 a 12 años; por otro lado, la periodontitis se presenta en $0,32 \%$ de los adolescentes de 15 a 19 años. En Nicaragua, la gingivitis se observado en un $77 \%$ de niños de 12 años y en 
un 68,5 \% en los de 15 años. En Panamá, la gingivitis se reporta en el $55 \%$ de la población de 6 a 19 años (11).

En Colombia, el tercer estudio nacional de salud bucal ENSAB III de 1998 encontró que el 7,3 $\%$ de los niños entre 7 y 12 años presentaba al menos un marcador periodontal: sangrado en el $51,9 \%$ de los niños de 7 años, mientras que sangrado y cálculos eran evidentes en el 60,9\% de los de 12 años (12). En contraste, el ENSAB IV de 2013-2014 informó que el 10,69 \% de los adolescentes colombianos presenta periodontitis agresiva; sin embargo, en el documento no se presentan datos que sustenten con precisión la prevalencia en niños menores de 12 años (13).

Las características clínicas y, en especial las microbiológicas, en la población pediátrica no se encuentran ampliamente documentadas. Las características microbiológicas presentes en los tejidos periodontales de niños y adolescentes difieren de la población adulta, razón por la cual Bimstein et al. (8) y Romero et al. (14) refieren que el surco gingival en los niños no es fácilmente colonizable.

El surco gingival es una de las estructuras del periodonto donde la microbiota es diversa y puede variar sustancialmente durante los cambios fisiológicos que experimentan niños y adolescentes. Tales cambios bacterianos contribuyen a una alteración del microambiente, los cuales también pueden presentarse en un periodonto joven con gingivitis o periodontitis (2). Por tratarse de condiciones inflamatorias e infecciosas, el incremento de periodontopatógenos en el surco gingival lleva al desarrollo de la gingivitis y la periodontitis. Es un factor determinante en la destrucción rápida e irreversible de los tejidos de sostén en un periodonto joven, al producirse 
bolsas periodontales, pérdida de inserción clínica, pérdida ósea, movilidad dental y pérdida de uno o varios dientes, lo que genera consecuencias biológicas, funcionales y estéticas (15).

Autores como Costalonga y Herzberg (16) hablan de una posible asociación entre la composición bacteriana del surco gingival y la presencia o ausencia de enfermedades gingivales y periodontales, que tienen en común los factores microbiológicos como principales implicados en el inicio y progresión de dichas patologías. A pesar de existir evidencia científica sobre la relación entre la composición bacteriana subgingival y la presencia o ausencia de enfermedad, en diferentes diseños y diversos grados de evidencia, la mayor parte de la información se refiere a la población adulta. Así, la información en población pediátrica es limitada e incluso desconocida $(17,18)$. Por lo tanto, buscar aclarar mediante el análisis de la evidencia existente la relación entre la composición bacteriana y el estado periodontal en niños/as hasta los 12 años de edad es relevante y útil para la práctica odontológica. En consecuencia, el objetivo de este estudio fue analizar la evidencia sobre la composición bacteriana en la población de 3 a 12 años y su relación con el estado gingival y periodontal.

\section{MATERIALES Y MÉTODOS}

Se realizó una revisión sistemática de la literatura con el fin de responder a la pregunta: En niños de 3 a 12 años, con dentición temporal y mixta, ¿qué relación tiene la composición bacteriana supra y subgingival con el estado periodontal? Los criterios de elegibilidad de la búsqueda incluyó publicaciones de 1 de enero de 1960 al 31 de diciembre de 2017 que presentaran hallazgos microbiológicos asociados al estado periodontal en niños de 3 a 12 años con dentición temporal y mixta, sin discriminación étnica ni sociodemográfica; estudios que vinculan 
personas con o sin compromiso sistémico, sin discriminación de idioma, cuyo diseño fuera meta-análisis, revisiones sistemáticas, observacional analítico de cohorte, casos y controles, y corte transversal. Para esta revisión no se establecieron criterios de exclusión. Las unidades de análisis que se determinaron fueron estado periodontal (salud y enfermedad), condición sistémica, asociaciones bacterianas, edad, condición sociodemográfica y género.

Se realizó una búsqueda electrónica en las bases de datos PubMed, SciELO, LiLACS, ProQuest, EBSCO, Web of Science, Scopus y ScienceDirect, así como una búsqueda manual para identificar la literatura disponible en publicaciones impresas. Los descriptores y conectores bolianos (en inglés) de la búsqueda fueron: bacteria AND pathogen OR microorganisms OR dental plaque OR biofilms OR microbiota oral OR microbiome AND gingival AND periodontal AND primary dentition AND mixed dentition AND subgingival AND supragingival AND child.

Dos investigadores iniciaron el proceso con la exclusión de artículos duplicados por medio del administrador bibliográfico EndNote. Se continuó con la lectura de títulos, seguido por la de los resúmenes seleccionados y de aquellos que cumplían con los criterios de elegibilidad definidos. Después se realizó la lectura de los textos completos. Las publicaciones seleccionadas se evaluaron a partir de su calidad metodológica por medio de los criterios de la lista de cotejo Strobe (individualizada para cada uno de los estudios analíticos). Luego, se identificaron los niveles de evidencia y los grados de recomendación de las publicaciones, según los criterios de la Scottish Intercollegiate Guidelines Network (SIGN). Se construyó una matriz bibliográfica con el fin de determinar las publicaciones que permitían responder a la pregunta de investigación y a los objetivos específicos del estudio (figura 1). La evaluación de sesgos se 
determinó a partir del tamaño de la muestra, la heterogeneidad de los estudios y los métodos estadísticos. Se realizó una lista de cotejo con 8 ítem. A 2 artículos no se les aplicó este filtro porque no cumplían con el patrón de análisis de la calidad metodológica.

FIGURA 1

FLUJOGRAMA

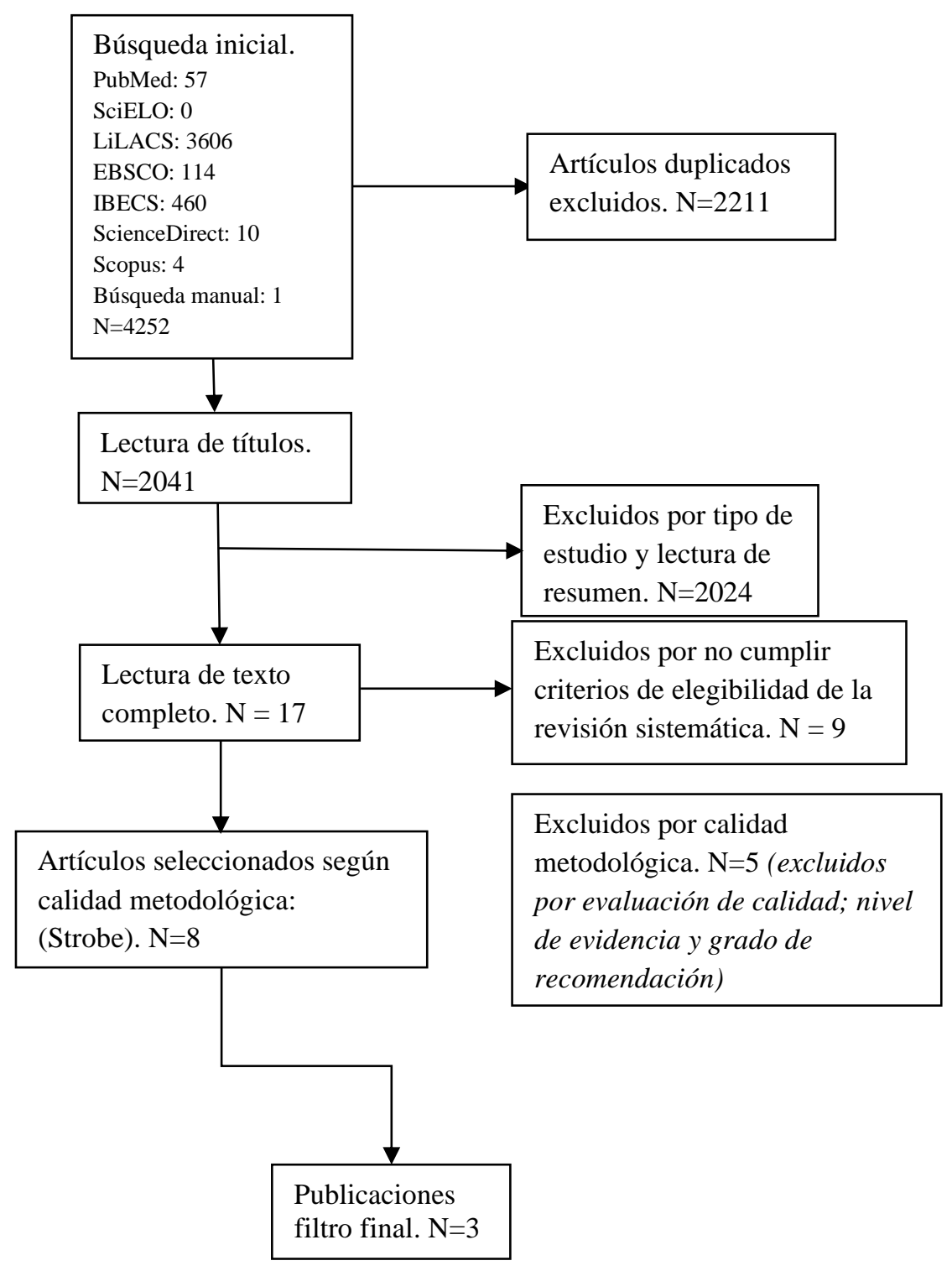




\section{RESULTADOS}

La búsqueda de artículos publicados entre el 1 de enero de 1960 y el 31 de diciembre de 2017 permitió identificar un total de 4252 títulos. Se excluyeron los duplicados y aquellos títulos que no se relacionaban con el tema de investigación. Después de la lectura de los resúmenes se excluyeron 2024 títulos. La lectura detallada de los textos completos de efectuó en 17 artículos de los cuales se excluyeron 9 que no cumplían los criterios de elegibilidad. Las 8 publicaciones restantes se evaluaron metodológicamente, por medio de las listas de cotejo. Se obtuvieron 5 artículos, de los que se excluyeron 2 después de la evaluación del nivel de evidencia y el grado de recomendación. La muestra final incluyó 3 publicaciones (figura 1).

A partir de las 3 publicaciones seleccionadas finalmente, se buscó determinar la relación entre la composición bacteriana supra y subgingival y el estado periodontal en niños de 3 a 12 años con dentición temporal y mixta (tabla 1). 
TABLA 1

ARTÍCULOS SELECCIONADOS FINALMENTE PARA EL ANÁLISIS

\begin{tabular}{|c|c|c|c|c|c|c|c|}
\hline Autor & $\begin{array}{c}\text { Diseño de } \\
\text { estudio }\end{array}$ & Muestra & Parámetros evaluados & Metodología & Resultados & Conclusiones & $\begin{array}{l}\text { NE \& } \\
\text { GR* }\end{array}$ \\
\hline $\begin{array}{l}\text { Papaioannou } \\
\text { et al., } 2009 \\
\text { (20) }\end{array}$ & 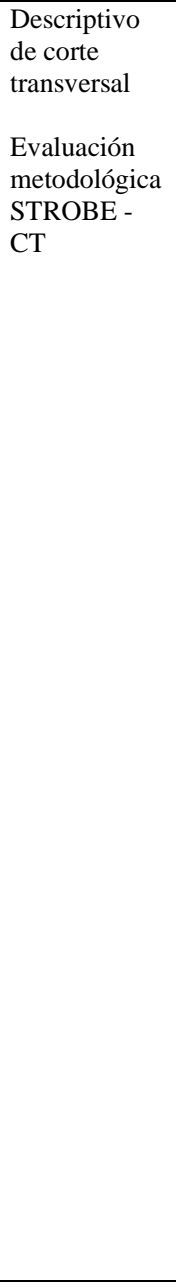 & $\begin{array}{l}\text { 93 niños: 3-12 } \\
\text { años } \\
\text { Grupos } \\
\text { etarios: } \\
3-6, \quad>6- \\
9, \quad>9-12 \\
\text { Cada grupo: } \\
\text { 31 niños }\end{array}$ & $\begin{array}{l}\text { Índice simplificado de placa } \\
\text { de O’Leary } \\
\text { Índice gingival simplificado } \\
\text { Examen clínico: Un } \\
\text { operador }\end{array}$ & $\begin{array}{l}\text { Identificación bacteriana por } \\
\text { técnica de hibridación } \\
\text { Detección de } 38 \text { especies } \\
\text { bacterianas } \\
5 \text { microambientes orales: } \\
\text { * Biopelícula Supragingival: } \\
\text { todos los dientes erupcionados } \\
\text { * Biopelícula Subgingival: Al } \\
\text { menos uno de los segundos } \\
\text { molares temporales o primeros } \\
\text { molares permanentes de niños > } \\
5 \text { años con dentición mixta } \\
\text { * Dorso de lengua } \\
\text { * Tejidos blandos } \\
\text { * Saliva }\end{array}$ & 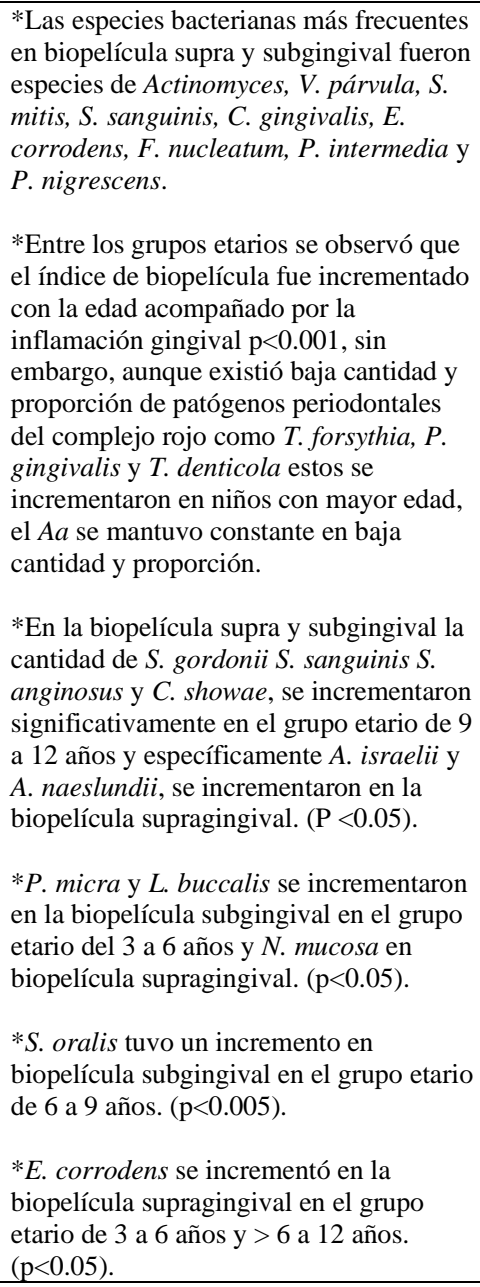 & $\begin{array}{l}\text { *Las diferencias en el perfil } \\
\text { entre los grupos de edad } \\
\text { sugieren una maduración de la } \\
\text { microbiota oral. } \\
\text { *A medida que la edad se } \\
\text { incrementa, } \\
\text { concomitantemente se } \\
\text { incrementó el nivel de } \\
\text { biopelícula bacteriana y la } \\
\text { inflamación gingival. } \\
\text { *Las bacterias pertenecientes } \\
\text { al complejo rojo (T. forsythia, } \\
P . \text { gingivalis y T. denticola), se } \\
\text { detectaron en todos los grupos } \\
\text { etarios en bajas proporciones, } \\
\text { sin embargo, se observó una } \\
\text { tendencia al incremento en el } \\
\text { grupo de mayor edad (> } 9 \text { a } 12) \\
\text { especialmente en biopelícula } \\
\text { subgingival. } \\
\text { *Los autores sugieren que el } \\
\text { incremento de la } P \text {. gingivalis } \\
\text { con la edad posiblemente esté } \\
\text { relacionado con el incremento } \\
\text { de la biopelícula bacteriana y } \\
\text { la inflamación gingival. }\end{array}$ & $2+\mathrm{C}$ \\
\hline $\begin{array}{l}\text { Sjödin et al., } \\
1995 \text { (21) }\end{array}$ & $\begin{array}{l}\text { Prevalencia } \\
\text { analítica } \\
\text { Evaluación } \\
\text { metodológica }\end{array}$ & $\begin{array}{l}\mathrm{N}=3896 \text { niños } \\
\text { de } 7-9 \text { años } \\
\text { Grupo estudio } \\
\text { (n=26 niños), } \\
\text { con evidencia }\end{array}$ & 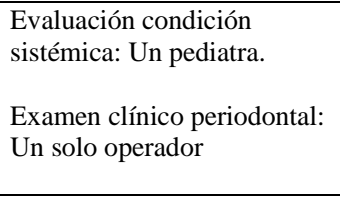 & $\begin{array}{l}\text { Examen físico (raquitismo, } \\
\text { hiperqueratosis, alteraciones piel } \\
\text { / ojos), análisis sanguíneo y } \\
\text { orina } \\
\text { Selección de sitio: }\end{array}$ & $\begin{array}{l}\text { *En } 26 \text { niños se presentó pérdida de } \\
\text { hueso (grupo estudio) y } 20 \text { sin pérdida de } \\
\text { hueso (grupo control), ninguno de los } \\
\text { niños presentó enfermedades sistémicas } \\
\text { aparentes. } 4 \text { niños con pérdida de hueso } \\
\text { y } 2 \text { controles presentaron otitis media en }\end{array}$ & $\begin{array}{l}\text { *En los niños con pérdida ósea } \\
>2 \text { superficies se identificó } A . \\
\text { actinomycetemcomitans a nivel } \\
\text { subgingival, indicando un } \\
\text { periodonto susceptible a }\end{array}$ & $2+\mathrm{C}$ \\
\hline
\end{tabular}




\begin{tabular}{|c|c|c|c|c|c|c|c|}
\hline & $\begin{array}{l}\text { STROBE - } \\
\text { CT }\end{array}$ & $\begin{array}{l}\text { radiográfica } \\
\text { de pérdida } \\
\text { ósea (en } 2 \text { o } \\
\text { más } \\
\text { superficies } \\
\text { proximales de } \\
\text { molares } \\
\text { deciduos) } \\
\text { Grupo control } \\
\text { (n=20 niños), } \\
\text { sin pérdida de } \\
\text { hueso }\end{array}$ & $\begin{array}{l}\text { Parámetros clínicos: } \\
\text { * Profundidad de bolsa } \\
\text { * Nivel de inserción clínica } \\
\text { * Sangrado y/o supuración } \\
\text { al sondaje } \\
\text { * Presencia o ausencia de } \\
\text { placa. } \\
\text { Examen radiográfico } \\
\text { Análisis microbiológico: A } \\
\text { partir de biopelícula } \\
\text { subgingival en dientes } \\
\text { primarios con mayor } \\
\text { profundidad de bolsa, } \\
\text { excepto en dientes en } \\
\text { proceso de } \\
\text { exfoliación/erupción en } \\
\text { donde se tomaba la segunda } \\
\text { bolsa más profunda. } \\
\text { Análisis de anticuerpos } \\
\text { séricos: Anti-Leucotoxina } \\
\text { A.a. }\end{array}$ & $\begin{array}{l}\text { *Profundidad de bolsa mayor } \\
\text { *Pérdida ósea marginal > } 2 \mathrm{~mm} \text {, } \\
\text { desde unión amelocementaria a } \\
\text { cresta ósea } \\
\text { Toma de muestra microbiológica } \\
\text { de biopelícula subgingival en } \\
\text { dientes primarios con mayor } \\
\text { profundidad de bolsa, excepto en } \\
\text { dientes en proceso de } \\
\text { exfoliación/erupción en donde se } \\
\text { tomó la segunda bolsa más } \\
\text { profunda. } \\
\text { Análisis microbiológico: } \\
\text { Biopelícula subgingival con } \\
\text { cultivo microbiológico selectivo } \\
\text { de A.a. TSBV } \\
\text { Análisis de anticuerpos séricos: } \\
\text { Anti-Leucotoxina A.a. mediante } \\
\text { ensayo de liberación } 51 C r\end{array}$ & $\begin{array}{l}\text { algún momento de su vida y uno } \\
\text { presentó un quiste del oído medio. } \\
\text { *Se logró identificar A.a. en } 14 \text { de } 26 \\
\text { niños con bolsa y pérdida de inserción } \\
\text { clínica, en la zona más profunda del } \\
\text { surco gingival. En ninguna muestra de } \\
\text { los controles se encontró este } \\
\text { microorganismo. }(\mathrm{P}<0.001) \text {. En } 22 / 26 \\
\text { del grupo caso y } 7 / 20 \text { del grupo control } \\
\text { se evaluó la presencia de anticuerpos } \\
\text { contra la leucotoxina de } A . a 11 / 22 \text { fueron } \\
\text { positivos y los } 7 \text { del grupo control fueron } \\
\text { negativos. }\end{array}$ & enfermedad periodontal. & \\
\hline $\begin{array}{l}\text { Kamma et al., } \\
2000(22)\end{array}$ & $\begin{array}{l}\begin{array}{l}\text { Prevalencia } \\
\text { analítica }\end{array} \\
\text { Evaluación } \\
\text { metodológica } \\
\text { STROBE - } \\
\text { CT }\end{array}$ & $\begin{array}{l}40 \text { niños } \\
\text { seleccionados } \\
\text { aleatoriamente } \\
\text { de escuelas } \\
\text { primarias } \\
\text { públicas y } \\
\text { privadas de } \\
\text { Atenas: } 20 \\
\text { niños y } 20 \\
\text { niñas. } \\
\text { Sistémicament } \\
\text { e sanos de } 7 \text { a } \\
8 \text { años con } \\
\text { dentición } \\
\text { mixta }\end{array}$ & $\begin{array}{l}\text { Examen clínico: Un solo } \\
\text { operador } \\
\text { Examen periodontal: } \\
\text { *Profundidad de bolsa } \\
\text { * Índice de placa y sangrado } \\
\text { de Löe } \\
\text { Análisis microbiológico: } \\
\text { muestras de placa } \\
\text { subgingival tomadas con } \\
\text { puntas de papel en } \\
\text { mesovestibular de } 21,41, \\
16,36,53,73,64 \text { y } 84 .\end{array}$ & $\begin{array}{l}\text { Cultivo microbiológico: } \\
\text { * Agar ETSA y KVLB-2 para } \\
\text { bacterias pigmentadas y no } \\
\text { pigmentadas } \\
\text { * Agar TSBV para A.a, agar } \\
\text { Wollinella para Campylobacter } \\
\text { rectus y } \text { C. concisus } \\
\text { * Agar NAM para } \text { B. forsythus } \\
\text { Para la identificación se } \\
\text { utilizaron los sistemas API } 20 \\
\text { STREP, RapID ANA II y API } \\
\text { ZYM }\end{array}$ & 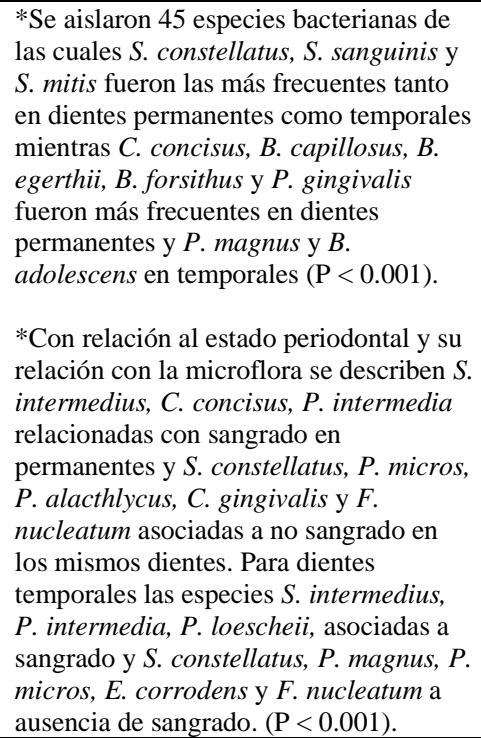 & $\begin{array}{l}\text { *Los niños a una edad } \\
\text { temprana con dentición mixta } \\
\text { albergan una bacteria } \\
\text { anaeróbica gramnegativa } \\
\text { indicando la presencia de } \\
\text { algunos patógenos } \\
\text { periodontales. } \\
\text { *Los niños colonizados } \\
\text { tempranamente con especies } \\
\text { periodontopatógenas pueden } \\
\text { tener un mayor riesgo de } \\
\text { presentar enfermedad } \\
\text { periodontal, ya sea en la } \\
\text { infancia o en la adultez. } \\
\text { *Los autores sugieren que la } \\
\text { Prevotella y Porphyromonas } \\
\text { spp. parecen relacionarse } \\
\text { secundariamente con el cambio } \\
\text { en el microambiente y una } \\
\text { tendencia incrementada al } \\
\text { sangrado }\end{array}$ & $2+C$ \\
\hline
\end{tabular}

*NE \& GR: Nivel evidencia y grados de recomendación 


\section{Composición bacteriana supra y subgingival en la población de 3 a 12 años y su relación con el estado gingival y periodontal}

Papaioannou et al. (19) establecieron que los perfiles microbianos de la biopelícula supra y subgingival son similares en más del $80 \%$ de las 38 especies analizadas. Predominan Actinomyces, V. parvula, S. mitis, S. sanguinis, C. gingivalis, E. corrodens, F nucleatum, P. intermedia y $P$. nigrescens. ( $\mathrm{p} \leq 0,001)$. Los mismos autores señalaron que microorganismos como $S$. gordonii, $S$. sanguinis, S. anginosus, y $C$. showae aumentan en proporción a la edad ( $\mathrm{p}<0,05)$, lo que sustenta la relación de la colonización temprana de la microbiota supra y subgingival con la presencia de inflamación gingival en niños de mayor edad (9-12 años) ( $p<0,001)$ (tabla 1).

De acuerdo con la edad y el tipo de microambiente, A. israelii y A. naeslundii incrementaron en la biopelícula supragingival en el grupo de 9-12 años (p < 0,05), así como P. micra y L. buccalis aumentaron en la biopelícula subgingival en el grupo de 3-6 años $(\mathrm{p}<0,05)$. Del mismo modo, E. corrodens se observó aumentado en biopelícula supragingival en los grupos de 3-6 y >6-9 años $(\mathrm{p}<0,05)$. Asimismo, $S$. oralis fue mayor en biopelícula subgingival en el grupo de >6-9 años ( $\mathrm{p}$ $<0,005)$, y $N$. mucosa en biopelícula supragingival en el grupo de 3-6 años $(\mathrm{p}<0,05)(19)$.

Por otra parte, Sjödin et al. (20) detectaron, en niños de 7-9 años, A. actinomycetemcomitans en la bolsa periodontal de dientes con pérdida de inserción clínica. En niños con periodonto sano, no se identificó el microorganismo ( $\mathrm{p}<0,001$ ) (tabla 1). De 22 niños a quienes se les realizaron pruebas de anticuerpos, la antileucotoxina de A. actinomycetemcomitans, 11 fueron positivos. 
En el tercer estudio, Kamma et al. (21) hallaron diferencias estadísticamente significativas ( $<$ < 0,001) con respecto a la composición de la microbiota subgingival de dientes deciduos y permanentes en niños de 7-8 años con dentición mixta (tabla 1). Las especies aisladas más frecuentemente en dientes permanentes y deciduos fueron $S$. constellatus, S. sanguinis (previamente, S. sanguis) y S. mitis, en más del $75 \%$. Las especies bacterianas que mostraron diferencias estadísticamente significativas en dientes permanentes fueron C. concisus, B. capillosus, B. egerthii, B. forsythus y $P$. gingivalis, mientras que en dientes deciduos fueron $P$. magnus, y $B$. adolescens $(\mathrm{p}<0,001)$ (tabla 1). El examen periodontal en dicho estudio lo realizó un solo operador calibrado quien registró la profundidad de la bolsa periodontal, el índice placa bacteriana y el sangrado en 6 sitios de dientes permanentes (21, 41, 16 y 36, según la nomenclatura dígito dos) y temporales $(64,84,53$ y 73, de acuerdo con la misma nomenclatura). Al analizar su relación con la microflora, describen $S$. intermedius, $C$. concisus y $P$. intermedia asociadas con el sangrado en dientes permanentes y $S$. constellatus, $P$. micros, $P$. alacthlycus, $C$. gingivalis y $F$. nucleatum asociados a la ausencia de sangrado en los mismos dientes. En cuanto a los dientes temporales, $S$. intermedius, $P$. intermedia y $P$. loescheii estaban asociadas con el sangrado, en tanto que $S$. constellatus, P. magnus, P. micros, E. corrodens y F. nucleatum en ausencia del mismo (p < 0,001).

Después del análisis de los 3 artículos que formaron parte de la muestra final se infiere que no hay evidencia científica sólida y suficiente sobre la relación del estado periodontal con la bacterias en la población de 3-12 años. 


\section{Relación entre la condición sistémica y el estado periodontal en la población de 3-12 años}

En el estudio de Sjödin et al. (20) se describen 6 casos de niños con y sin pérdida de inserción y que habían sufrido de otitis media en algún momento de su vida. Uno de los casos habla de la presencia de un quiste del oído medio al momento de la toma de la muestra. En dicha investigación no se encontró asociación entre la otitis media y el quiste del oído medio con el estado periodontal de la población de 3-12 años (tabla 1).

Kamma et al. (21) incluyen pacientes sistémicamente sanos para realizar una investigación sobre el perfil de la microbiota subgingival en niños con dentición mixta. Sin embargo, no mencionan en sus hallazgos la relación de la condición sistémica con la composición de microbiota del surco gingival. La evidencia encontrada sobre la condición sistémica y el estado periodontal no es concluyente (tabla 1).

\section{Relación de la condición sociodemográfica y el estado periodontal en niños de 3-12 años} La limitación para analizar la información sociodemográfica y el estado periodontal es evidente. En las publicaciones analizadas en este estudio se mencionaron aspectos relacionados con la edad, el género, el nivel socioeconómico, la escolaridad y la procedencia. Sin embargo, la información se redujo a la exposición de datos descriptivos sin establecer alguna asociación entre las variables. La escolaridad de los padres no fue analizada en ninguna de las publicaciones.

\section{Otros hallazgos}

Papaioannou et al. (22) describen el aumento del índice de placa con la edad, así como la inflamación gingival $(\mathrm{p}<0,001)$. Asimismo, los patógenos periodontales incrementan con la edad (tabla 1). 


\section{DISCUSIÓN}

La interacción del microbioma en la cavidad oral se ha descrito ampliamente a lo largo de los años, desde su efecto protector hasta la generación de enfermedades dentales y periodontales. Se reconocen especies bacterianas que están relacionadas con diferentes condiciones clínicas. La disbiosis bacteriana favorece la presencia de colonizadores tardíos como $P$. gingivalis, $T$. forsythia y P. intermedia, los cuales se han relacionados con el desarrollo de patologías periodontales (23). La composición bacteriana de la biopelícula subgingival asociada con gingivitis y periodontitis resulta de las interacciones dinámicas con el microambiente (24). Los tejidos periodontales sanos o en presencia de patología gingival/periodontal, en la población pediátrica, presentan características diferentes respecto al periodonto de los adultos en su aspecto clínico, histológico y microbiológico (25). Godoy et al. (16) determinaron que las características microbiológicas en la población pediátrica no se encuentran ampliamente documentadas. Por otro lado, Nobre et al. (26) identificaron microorganismos que están presentes en adultos pero no en niños con periodonto sano; sin embargo, se modifican con el cambio de la dentición temporal a mixta por variaciones en el microambiente.

El objetivo de la presente revisión sistemática fue analizar la composición bacteriana supra y subgingival en población de 3-12 años y su relación con el estado gingival y periodontal. La frecuencia global de gingivitis en niños mayores de 7 años es del $70 \%$, en tanto que la prevalencia de enfermedad periodontal destructiva es baja, esto es, $0,9 \%$ a $4,5 \%$ (7). Los resultados de esta revisión sistemática identificaron, en el estudio de Sjödin et al. (20), A. 
actinomycetemcomitans en el surco gingival de niños de 7-9 años, sistémicamente sanos, con pérdida de inserción y parámetros clínicos variables. Por otro lado, en niños con periodonto sano no se halló este microorganismo. Cabe resaltar como limitante de ese estudio que únicamente realizaron análisis microbiológico para la identificación de $A$. actinomycetemcomitans. Morinushi et al. (27), en un estudio sobre gingivitis en niños y la colonización de $P$. gingivalis y A. actinomycetemcomitans, encontraron niveles detectables de $P$. gingivalis en más del $60 \%$ y $75 \%$ de A. actinomycetemcomitans en los niños examinados y asociados con niveles mayores de inflamación gingival, sin mostrar diferencias estadísticamente significativas entre los grupos. Asimismo, hallaron que había una relación inversa entre la presencia de anticuerpos séricos para $P$. gingivalis relacionado con el índice de enfermedad periodontal y de pérdida de inserción proximal independiente de la edad $(\mathrm{p}<0,03)$. Por otra parte, encontraron una relación directa para A. actinomycetemcomitans. Los mismos autores establecieron que el A. actinomycetemcomitans se relacionó significativamente con el índice de enfermedad periodontal en el grupo de mayor edad $(\mathrm{p}<0,03)(28)$. También mostraron una asociación del alto riesgo a presentar pérdida de inserción en niños y adolescentes con enfermedad periodontal destructiva en pacientes portadores del clon JP2 del A. actinomycetemcomitans, mientras que los portadores del clon no-JP2 tuvieron un riesgo reducido a presentar periodontitis, lo que sugiere una competencia ecológica en el nicho.

En el presente estudio, se encontró que en el estudio de Papaioannou et al. (19), al analizar distintos microambientes orales en 93 niños sistémica y periodontalmente sanos, de 3 a 12 años de edad, había similitud en más del $80 \%$ de la composición bacteriana entre la biopelícula supra y subgingival. Se analizaron mediante prueba de hibridación de ADN 38 especies de bacterias orales 
y se identificaron altas frecuencias de Actinomyces, V. párvula, S. mitis, S. sanguinis, C. gingivalis, E. corrodens, F. nucleatum, P. intermedia y P. nigrescens. Estos resultados eran esperados, de acuerdo con la secuencia de formación de la biopelícula, puesto que la mayoría de estas bacterias son aerobias o anaerobias facultativas asociadas a estados saludables. Tal fue el caso de Capnocytophaga y Actinomyces. Por otra parte, la frecuencia y proporción de bacterias como A. actinomycetemcomitans, C. rectus, T. forsythia, T. denticola y P. melaninogenica fue baja. Estos resultados concuerdan con otros estudios como el referenciado por Darby y Curtis (29), el cual sugiere que el A. actinomycetemcomitans es capaz de establecerse en la cavidad oral en niños de 57 años con dientes permanentes recién erupcionados.

En el estudio realizado por Papaioannou et al. (20) se identificaron $P$. gingivalis y $P$. micra en los 5 microambientes muestreados. No obstante, Asikainen et al. (30) describieron que la frecuencia de $P$. gingivalis fue menor al $5 \%$ en niños de 5-10 años periodontalmente sanos. Cabe resaltar que la presencia de microorganismos periodontopatógenos en esta población o con gingivitis es actualmente debatida debido a que autores como Tanaka et al. (31) sugieren que la presencia de este tipo de bacterias puede estar de forma transitoria en la cavidad oral sin generar signos clínicos que sugieran enfermedad. Sin embargo, otros autores como Bimstein et al. (32) sugieren que la presencia de bacterias detectadas desde temprana edad podría facilitar a largo plazo el establecimiento de enfermedad periodontal. Estos autores mencionan el gran impacto que podría tener al prevenir la enfermedad periodontal en niños durante la dentición primaria y mixta sobre la reducción en la severidad y progresión de dicha enfermedad en población adolescente y adulta. 
Kamma et al. (21) observaron en una muestra de 40 niños de 7-8 años, sistémicamente sanos, con dentición mixta, el análisis de las diferencias entre la microbiota en dientes deciduos (53, 73, 64, 84, clasificación dígito dos) y permanentes $(21,41,16,36)$. Identificaron que las especies bacterianas más frecuentemente aisladas tanto en dientes permanentes como deciduos fueron $S$. constellatus, S. sanguinis y S. mitis. Sin embargo, las que mostraron diferencias estadísticamente significativas en dientes deciduos en relación con los dientes permanentes fueron $P$. magnus, y B. adolescens; mientras que $C$. concisus, B. capillosus, B. egerthii y $P$. gingivalis fueron más frecuentes en dientes permanentes.

La comparación de bacterias catalogadas como periodontopatógenas entre dientes permanentes y dientes deciduos mostró: $P$. gingivalis $12,5 \%$ vs. 8,8 \%; P. intermedia 42,5 \% vs. $35 \%$; $C$. gingivalis $46,3 \%$ vs. $33,8 \% ; F$. nucleatum $37,5 \%$ vs. $33, .8 \%$ (21). Otros autores que describen diferencias entre dientes temporales y deciduos, como Shi et al. (33), describen que la microbiota de niños de 10-12 años mediante secuenciación del gene 16S rRNA. Observaron que dicha composición fue similar entre molares y caninos deciduos y entre molares e incisivos permanentes. Adicionalmente, identificaron que las diferencias significativas en la composición de la comunidad microbiana en el perfil supragingival se debieron principalmente a la presencia de Actinomyces en dientes permanentes y de Treponema en dientes deciduos. En contraste, Okada et al. (22) estudiaron una muestra de 24 niños clínicamente sanos, 83 con gingivitis y 12 con periodontitis. A partir de muestras de biopelícula bacteriana identificaron 5 periodontopatógenos $(P$. intermedia, $T$. denticola, B. forsythus, P. nigresens y C. rectus). Al examen clínico observaron 36 niños con dentición primaria y 83 con dentición mixta. C. rectus se logró identificar en todos los niños, independientemente del tipo de dentición y del diagnóstico clínico. $P$. nigrescens fue el segundo 
microorganismo más frecuente en 18 de los 36 niños con dentición primaria y en 66 de los 83 niños con dentición mixta. Los resultados para B. forsythus fue de 14 de los 36 niños con dentición primaria y de 54 de los 83 niños con dentición mixta. Para T. denticola, 4 de los 36 niños con dentición primaria fueron positivos para este microorganismo al igual que 13 de los 83 con dentición mixta. Por último, $P$. intermedia se logró identificar en 2 de los 36 niños con dentición primaria y 6 de los 83 niños con dentición mixta. Se puede resaltar que, para el grupo de niños con dentición mixta, $P$. nigrescens presentó una frecuencia del 79,5\% en contraste con los niños con dentición primaria en quienes fue del $50 \%$.

En relación con lo anterior, Bimstein et al. (34) indicaron un incremento significativo de $P$. gingivalis en la biopelícula subgingival de dientes permanentes mayor que dientes deciduos ( $\mathrm{p}$ = 0,006) (26). Similar a los resultados de la presente revisión, Kamma et al. (1) refirieron que las especies bacterianas que mostraron diferencias estadísticamente significativas con un incremento en dientes permanentes con relación a dientes deciduos fueron $P$. gingivalis, $C$. concisus, B. capillosus, B. egerthii, y B. forsythus. En esta unidad de análisis no fue posible realizar interpretaciones adicionales debido a la heterogeneidad de la composición de la microflora de dientes permanentes y deciduos. Por su parte, Lamell et al. (35), mediante un análisis de regresión logística, hallaron que no existió relación entre la edad y la colonización de $P$. gingivalis o A. actinomycetemcomitans $(\mathrm{p}=0,53)$. Las bacterias pertenecientes al complejo rojo, como T. forsythia, P. gingivalis y $T$. denticola, se detectaron en todos los grupos etarios en bajas proporciones; no obstante, mostraron una tendencia a incrementar en el grupo de mayor edad (>9-12 años), especialmente en biopelícula bacteriana subgingival (19). Resultados similares fueron reportados por Lamell et al. (35), quienes encontraron frecuencias 
bajas de dicha bacteria en niños en de 7-11 años e incrementaron considerablemente en adultos jóvenes (17-22 años). Por su parte, Okada et al. (22) observaron que la frecuencia de $B$. forsythus y $P$. nigresens se incrementó a medida que aumentó la edad de los niños.

Con respecto al género, autores como Bimstein et al. (34) encontraron en población sistémicamente sana de 11-15 años, valores mayores de $P$. gingivalis en niñas (media $=$ $\left.2.2 \mathrm{X} 10^{5} ; \mathrm{DS}=4.6 \mathrm{X} 10^{4}\right)$ al compararlas con los niños $\left(\right.$ media $\left.=4.7 \mathrm{X} 10^{4} ; \mathrm{DE}=4.2 \mathrm{X} 10^{4}\right)$. Por otra parte, citan a Celeste et al., quienes no hallaron relación entre etnia (afroamericanos, caucásicos y otras razas) o sexo y la colonización con A. actinomycetemcomitans o $P$. gingivalis en población pediátrica de 0 a 8 años.

En las publicaciones seleccionadas para el análisis de la presente investigación, no se evidencia el estudio de las principales condiciones sociodemográficas y de determinantes sociales, relacionadas al estado periodontal de la población.

La evidencia analizada en este estudio sobre la condición sistémica, la composición microbiana y el estado periodontal no fueron concluyentes, ya que las unidades de análisis no incluyeron información suficiente debido a la gran heterogeneidad de condiciones sistémicas que presentaban las poblaciones y los diferentes diseños de estudio que no permitieron efectuar un mayor análisis de estas condiciones. El único estudio que intenta relacionar los elementos de esta unidad de análisis en la población pediátrica fue el de Sjödin et al. (20) en el que se analizó una población de 3896 niños de 7-9 años. Encontraron en 32 niños pérdida ósea y de inserción del 0,8\%; ningún niño presentó enfermedades sistémicas evidentes, y hallaron la presencia de 
A. actinomycetemcomitans en 14 de los 26 niños en los sitios más profundos de los sitios con pérdida de inserción. Sin embargo, 6 niños refirieron otitis media y un caso de quiste del oído medio, lo cual no pudo relacionarse con su condición periodontal. Por su parte, Amano et al. (36) establecieron que $C$. ochracea, C. sputigena, C. rectus, E. corrodens y $A$. actinomycetemcomitans presentaron mayor frecuencia en niños con síndrome de Down de 2-4 años. Yamazaki et al. (37) coinciden con la investigación anteriormente mencionada, identificando una mayor tasa de detección de microorganismos periodontopatógenos en pacientes con síndrome de Down en comparación con niños sin esta condición; sin embargo, solo se observó diferencia estadística en $C$. rectus $(\mathrm{p}<0,01)$.

Posterior al análisis de cada uno de los artículos seleccionados, no se identificó suficiente evidencia científica que proporcionara información sobre la relación del estado periodontal con los tipos de bacterias en la población pediátrica de 3-12 años. Lo anterior se explicó por las publicaciones de la interacción bacteriana en la población adulta, mientras que en la población pediátrica la información es limitada. No obstante, se sugiere que a medida que se incrementa la edad, se favorece la presencia de patógenos periodontales relacionados con la presencia de enfermedad periodontal.

Las limitaciones de la presente investigación se relacionan con la dificultad para comparar las diferentes publicaciones debido a la heterogeneidad de los estudios y a las características metodológicas evidenciadas en la literatura analizada. Finalmente, algunas publicaciones no cumplían con los estándares de la lista de cotejo para su clasificación, según las guías de evaluación metodológica. 


\section{CONCLUSIONES}

No se identificó suficiente evidencia científica que sustentara la relación entre la composición bacteriana con el estado gingival y periodontal en niños de 3 a 12 años. Se sugiere que a medida que incrementa la edad se favorece la presencia de patógenos periodontales relacionados con la presencia de enfermedad periodontal.

El perfil microbiano supra y subgingival tienen un alto porcentaje de similitud.

En relación con la condición sociodemográfica, la sistémica, la composición microbiana y el estado periodontal, los hallazgos de esta investigación establecen una limitación relacionada con la información disponible a esta relación.

No se identificó evidencia científica suficiente que proporcionara información sobre la relación del estado periodontal con la interacción entre bacterias, en la población pediátrica de 3-12 años.

\section{RECOMENDACIONES}

Se recomienda realizar estudios clínicos observacionales con componente analítico que incluyan análisis microbiológicos y su relación con estado periodontal en niños con dentición decidua. 
Realizar estudios específicos de condición sistémica y su relación con la composición bacteriana y el estado periodontal en niños.

\section{AGRADECIMIENTOS}

La presente investigación se realizó con recursos propios de los investigadores.

\section{REFERENCIAS}

1. Kamma JJ, Diamanti-Kipioti A, Nakou M, Mitsis FJ. Profile of subgingival microbiota in children with primary dentition. J Periodontal Res. 2000 Feb; 35(1): 33-41; https://orcid.org/10.1034/j.1600-0765.2000 035001033.x

2. Cruz Quintana SM, Diaz Sjostrom P, Arias Socarrás D, Mazón Baldeón GM. Microbiota de los ecosistemas de la cavidad bucal. Rev Cub Estomatol. 2017 Ene; 54(1): 84-89.

3. Crossner CG, Carlsson J, Sjödin B, Tarnvik A, Unell L, Venge P, Wranne L. Periodontitis in the primary dentition associated with Actinobacillus actinomycetemcomitans infection and leukocyte dysfunction. A 3 1/2 year follow-up. J Clin Periodontol. 1990 Apr; 17(4): 264-7. http://doi.org/10.1111/j.1600-051x.1990.tb00023.x

4. Botero JE, Rösing CK, Duque A, Jaramillo A, Contreras A. Periodontal disease in children and adolescents of Latin America. Periodontol 2000. 2015; 67(1): 34-57. http://doi.org/10.1111/prd.12072 
5. Gamboa F, Garcia DA, Acosta A, Mizrahi D, Paz A, Martinez D, Arévalo A, Aristizábal F, Abba M. Presence and antimicrobial profile of gram-negative facultative anaerobe rods in patients with chronic periodontitis and gingivitis. Acta Odontol Latinoam. 2013; 26(1): 24-30.

6. Gamboa F, Acosta A, Garcia DA, Velosa J, Araya N, Ledergerber R. Occurrence of Porphyromonas gingivalis and its antibacterial susceptibility to metronidazole and tetracycline in patients with chronic periodontitis. Acta Odontol Latinoam. 2014; 27(3): 137-144. http://doi.org/10.1590/S1852-48342014000300007

7. Albandar JM, Rams TE. Risk factors for periodontitis in children and young persons. Periodontol 2000. 2002 Apr; 29(1): 207-22. http://doi.org/10.1034/j.1600-0757.2002. $\underline{290110 . x}$

8. Bimstein E, Lustmann J, Soskolne WA. A clinical and histometric study of gingivitis associated with the human deciduous dentition. J Periodontol. 1985 May; 56(5): 293-6. http://doi.org/10.1902/jop.1985.56.5.293

9. Elias-Boneta AR, Ramirez K, Rivas-Tumanyan S, Murillo M, Toro MJ. Prevalence of gingivitis and calculus in 12-year-old Puerto Ricans: a cross-sectional study. BMC Oral Health. 2018 Jan; 18(1): 13. http://doi.org/10.1186/s12903-017-0471-5

10. Linares Vieyra C, Martínez de Jesús C, González Guevara M, Murrieta Pruneda J. Prevalencia de gingivitis y su relación con factores de riesgo en niños. Salud (i) Ciencia. 2015; 21(2): 142-147.

11. Kinane DF, Hodge PJ. Periodontal disease in children and adolescents: introduction and classification. Periodontol 2000. 2001 Jun; 26(1): 7-15. http://doi.org/10.1034/j.1600$\underline{0757.2001 .2260101 . x}$ 
12. República de Colombia, Ministerio de Salud, Dirección General de Promoción y Prevención. III Estudio Nacional de Salud Bucal y II Estudio de factores de riesgo de las enfermedades crónicas. Bogotá, Colombia: Ministerio de Salud; 1999. https://www.visitaodontologica.com/ARCHIVOS/ARCHIVOSNORMAS/Salud\%20Publica_P_y_P/II_ESTUDIO_NACIONAL_SALUD_BUCAL.pdf

13. República de Colombia, Ministerio de Salud. IV Estudio Nacional de Salud Bucal ENSAB IV. Situación en salud bucal. Bogotá, Colombia: Ministerio de Salud; 2015. https://www.minsalud.gov.co/sites/rid/Lists/BibliotecaDigital/RIDE/VS/PP/ENSAB-IV$\underline{\text { Situacion-Bucal-Actual.pdf }}$

14. Romero M, Colmenares M, Benítez A, Baltodano F, Pacheco B. Diferencias histológicas en la encía de niños y adultos jóvenes con gingivitis inducida por biopelícula. Univ Odontol. 2011 Jul-Dic; 30(65): 79-88.

15. Laudenbach JM, Simon Z. Common dental and periodontal diseases: Evaluation and management. Med Clin North Am. 2014 Nov; 98(6): 1239-1260. http://doi.org/10.1016/j.mcna.2014.08.002

16. Costalonga M, Herzberg MC. The oral microbiome and the immunobiology of periodontal disease and caries. Immunol Lett. 2014 Dec; 162(200): 22-38. http://doi.org/10.1016/j.imlet.2014.08.017

17. Zhang Y, Wang X, Li H, Ni C, Du Z, Yan F. Human oral microbiota and its modulation for oral health. Biomed Pharmacother. $2018 \quad$ Mar; 99: 883-93. http://doi.org/10.1016/j.biopha.2018.01.146

18. Bimstein E, Matsson L. Growth and development considerations in the diagnosis of gingivitis and periodontitis in children. Pediatr Dent. 1999 May-Jun; 21(3): 186-91. 
19. Papaioannou W, Gizani S, Haffajee AD, Quirynen M, Mamai-Homata E, Papagiannoulis L. The microbiota on different oral surfaces in healthy children. Oral Microbiol Immunol. 2009 Jun; 24(3): 183-189. http://doi.org/10.1111/j.1399-302X.2008.00493.X

20. Sjödin B, Arnrup K, Matsson L, Wranne L, Carlsson J, Hanstrom L. Periodontal and systemic findings in children with marginal bone loss in the primary dentition. J Clin Periodontol. 1995 Mar; 22(3): 214-224. http://doi.org/10.1111/j.1600-051x.1995.tb00137.x

21. Kamma JJ, Diamanti-Kipioti A, Nakou M, Mitsis FJ. Profile of subgingival microbiota in children with mixed dentition. Oral Microbiol Immunol. 2000 Apr; 15(2): 103-111. http://doi.org/10.1034/j.1399-302x.2000.150206.x

22. Okada M, Hayashi F, Nagasaka N. PCR detection of 5 putative periodontal pathogens in dental plaque samples from children 2 to 12 years of age. J Clin Periodontol. 2001 Jun; 28(6): 576-582. http://doi.org/10.1034/j.1600-051x.2001.028006576.x

23. Colombo APV, Tanner ACR. The role of bacterial biofilms in dental caries and periodontal and peri-implant diseases: a historical perspective. J Dent Res. 2019 Apr; 98(4): 373-385. http://doi.org/10.1177/0022034519830686

24. Lang NP, Bartold PM. Periodontal health. J Clin Periodontol. 2018 Jun; 45(20): S9-S16. http://doi.org/10.1111/jcpe.12936.

25. Bimstein E, Matsson L, Soskolne AW, Lustmann J. Histologic characteristics of the gingiva associated with the primary and permanent teeth of children. Pediatr Dent. 1994 May-Jun; 16(3): 206-10.

26. Nobre CM, Fernandes-Costa AN, de Melo Soares MS, Pugliesi DM, de Vasconcelos Gurgel BC. Periodontal disease detection in primary and mixed dentitions. Eur Arch Paediatr Dent. 2016 Oct; 17(5): 407-411. http://doi.org/10.1007/s40368-016-0248-6 
27. Morinushi T, Lopatin DE, Van Poperin N, Ueda Y. The relationship between gingivitis and colonization by Porphyromonas gingivalis and Actinobacillus actinomycetemcomitans in children. J Periodontol. 2000 Mar; 71(3): 403-409. http://doi.org/10.1902/jop.2000.71.3.403

28. Haubek D, Ennibi OK, Poulsen K, Vaeth M, Poulsen S, Kilian M. Risk of aggressive periodontitis in adolescent carriers of the JP2 clone of Aggregatibacter (Actinobacillus) actinomycetemcomitans in Morocco: a prospective longitudinal cohort study. Lancet 2008 Jan; 371(9608): 237-242. http://doi.org/10.1016/S0140-6736(08)60135-X

29. Darby I, Curtis M. Microbiology of periodontal disease in children and young adults. Periodontol 2000. 2001 Jun; 26(1): 33-53. http://doi.org/10.1034/j.1600$\underline{0757.2001 .2260103 . x}$

30. Asikainen S, Chen C, Alaluusua S, Slots J. Can one acquire periodontal bacteria and periodontitis from a family member? J Am Dent Assoc. 1997 Sep; 128(9): 1263-1271. http://doi.org/10.14219/jada.archive.1997.0403

31. Tanaka S, Yoshida M, Murakami Y, Ogiwara T, Shoji M, Kobayashi S, Watanabe S, Machino M, Fujisawa S. The relationship of Prevotella intermedia, Prevotella nigrescens and Prevotella melaninogenica in the supragingival plaque of children, caries and oral malodor. J Clin Pediatr Dent. 2008 Spring; 32(3): 195-200. http://doi.org/10.17796/jcpd.32.3.vp65717781561811

32. Bimstein E, Huja PE, Ebersole JL. The potential lifespan impact of gingivitis and periodontitis in children. J Clin Pediatr Dent. 2013 Winter; 38(2): 95-99. http://doi.org/10.17796/jcpd.38.2.j525742137780336 
33. Shi W, Qin M, Chen F, Xia B. Supragingival microbial profiles of permanent and deciduous teeth in children with mixed dentition. PLoS One. 2016; 11(1): e0146938. https://doi.org/10.1371/journal.pone.0146938

34. Bimstein E, Ram D, Irshied J, Naor R, Sela MN. Periodontal diseases, caries, and microbial composition of the subgingival plaque in children: a longitudinal study. ASDC J Dent Child. 2002 May-Aug; 69(2): 133-7, 123.

35. Lamell CW, Griffen AL, McClellan DL, Leys EJ. Acquisition and colonization stability of Actinobacillus actinomycetemcomitans and Porphyromonas gingivalis in children. J Clin Microbiol. 2000 Mar; 38(3): 1196-1199.

36. Amano A, Kishima T, Kimura S, Takiguchi M, Ooshima $\mathrm{T}$, Hamada $\mathrm{S}$, et al. Periodontopathic bacteria in children with Down syndrome. J Periodontol. 2000 Feb; 71(2): 249-55. http://doi.org/10.1902/jop.2000.71.2.249

37. Yamazaki-Kubota T, Miyamoto M, Sano Y, Kusumoto M, Yonezu T, Sugita K, Okuda K, Yakushiji M, Ishihara K. Analysis of matrix metalloproteinase (MMP-8 and MMP-2) activity in gingival crevicular fluid from children with Down's syndrome. J Periodontal Res. 2010 Apr; 45(2): 170-176. http://doi.org/10.1111/j.1600-0765.2009.01214.x 